\section{Ethics consultation: a practical guide}

John LaPuma and David Schiedermayer, Boston, MA, Jones and Bartlett, 1993, 248 pages, $\$ 29.95$

\section{The health care ethics consultant}

Edited by Francoise E Baylis, Totowa, NJ, Humana Press, 1994, 221 pages, $\$ 39.50 \mathrm{hb}$

The field of bioethics has spawned sub-specialties during the past two decades, one of which, clinical ethics, has only recently received the attention it deserves. A comprehensive literature now exists to address particular problems of the clinician-patient encounter, scholarly journals have emerged, and professional associations have been created, whose focus is on the provision of clinical ethics 'consultation'. As befits a new specialty, healthy debate is occurring about what the specialty consists in and who ought to be qualified to engage in it. These two books, while not the first in this emerging specialty, provide readers with important insights not only into many of the substantive issues in clinical (or health care) ethics consultation, but also into the nature of the professional debate itself.

Ethics Consultation: A Practical Guide offers an approach to clinical ethics consultation which the authors believe will 'improve patient care'. It consists of four chapters, each devoted to a separate topic: case consultation; training, skills and certification; setting up practice, and consultants and committees. Many topics and issues are addressed in an easy-toread format. Important, practical issues rarely (or never) treated elsewhere in the literature are given an airing here, including: writing job descriptions; the differences between practice settings (urban $\mathrm{v}$ rural; teaching v community hospitals); management of personal stress, and the role of ethicists on clinical ethics and other institutional committees. An appendix of illustrative case consultations provides some insight into how the authors see ethics consultation occurring. A modest annotated bibliography is prepared for each chapter. Thirty-nine references are given.

For those readers already familiar with one of the authors' (JL) regular contributions to the clinical ethics literature, this book will have few surprises. It makes clear the authors' preferences that clinical ethics consultants should, ideally, be physicians capable of understanding medical terminology, performing physical examinations, and checking test results and drug prescriptions and the like. 'This is a book' they write, 'about a specific consultation method with actual patient care examples'. They are not apologists for this view, taking care to explain (unconvincingly in my view) why ethics consultants should be medical people. The argument is somewhat thin but is available, in that much of the empirical literature used to support this claim is written by one or both of the authors (and found in the bibliography). This is typical of the book, where controversial and currently debatable topics are presented as being resolved: the ethics consultant's primary activity is to resolve ethical dilemmas in patient care; clinical ethics constitutes a distinct professional activity; ethics consultants should have specific credentials/qualifications; all patients should be seen (and preferably examined) by an ethics consultant; consultations should proceed with the agreement of the attending physician, and consultations should be recorded in the patient's progress notes. Interestingly, there is an absence of discussion about what clinical ethicists ought to call themselves ('consultant', 'ethicist, 'clinical ethicist') and how professional titles ought to be used (since this affects perceptions of credibility).

Despite the caveat that the book is meant to be a practical guide focused 'primarily on the clinical model of ethics consultation', it is still somewhat frustrating to read about clinical ethics consultation constantly through the eyes of the physician-ethicist consultant. Issues of specialization, professionalism, and credibility are framed in such a way that the standard against which one ought to be judged is that of a medical doctor alone. Moreover, the context is decidedly American, although with effort it can be applied to other health care systems. It is worth noting that this approach has already been applied at the policy level. At the time of writing (Autumn 1994), the Illinois State Medical Society was considering resolution 3B (A-93), which would amend its constitution and bylaws to 'request the American Medical Association to urge the Health Insurance Association of America
(HIAA), the Health Care Financing Administration (HCFA), managed $\stackrel{\unrhd}{2}$ care organizations, and other third $m$ party payers to reimburse physician $\overrightarrow{\overrightarrow{0}}$. ethicists for ethics consultations'.

One-third of the written text is devoted to 25 illustrative cases, in $\stackrel{P}{\rightarrow}$ which the authors use the 'case presentation' rather than 'phenomeno- $\frac{\partial}{6}$ logical' tradition. This allows for more $\overline{\bar{N}}$ cases treated with brevity (which they readily admit to be a goal), but lacks an essential feature for those who read $\%$ the cases without the benefit of having $\overrightarrow{0}$ been there: how did they reason through these problems? It is regret- $\vec{\omega}$ table then that 'most of the clinical $\stackrel{\Omega}{\rightarrow}$ particulars, moral reasoning and ethical analyses are not recounted'. N

This text is likely to be of value $\cdot$ to physicians contemplating career changes or career enhancement to $\mathrm{N}$ incorporate ethics consultation into their practice. But I suspect it will also 의 be of value for those who remain $\rightarrow$ unconvinced that ethics consultation is a protected sub-specialty of medicine and want to read a clear $\frac{5}{2}$ description of that position in order to understand their own perceptions about this field.

In many respects, The Health Cooke Ethics Consultant provides a muce needed balance to the approach in the La Puma and Schiedermayer text. It is not the polar extreme; (which would have been a text written by two philosophers and theologians arguing that the only person capable of providing ethics consultation in hospital is someone philosophically trained? with some additional observational experience). The principal difference $\frac{\vec{\sigma}}{\delta}$ between this book and LaPuma and Schiedermayer's is that it is the direct 3 . result of a multidisciplinary research $\delta$ project designed to answer important 3 questions about what a health care 0 ethics consultant ought to look like. Each of fourteen authors from $\rightarrow$ different disciplinary backgrounds participated in a series of meetings, $N$ one of the results of which were the seven chapters in this book. N While the primary objective of this $\mathrm{C}_{\mathrm{J}}$ book is similar to LaPuma and $\sigma$ Schiedermayer's ('... to focus on an immediate practical problem: the role and responsibilities, the education $\$$ and training, and the certification and accreditation of health care ethics consultants ...'), it presumes that $\frac{}{\circ}$ health care ethics consultation (a term $\stackrel{\odot}{\stackrel{P}{~}}$ the authors prefer to 'ethics consul- $\stackrel{\mathbb{Q}}{\varrho}$ tant' or 'clinical ethicist') is, by its $\sigma$ nature, multidisciplinary. The book begins, then, with a different set of 
premises about ethics consultation from LaPuma and Schiedermayer's: namely that while the 'professionalisation' of ethics consultation may be an inevitability, there is still room to debate the advantages and disadvantages of certification - a point made quite clearly in Susan Sherwin's opening chapter (chapter 1).

There are other differences as well. This text is written by a group of scholars working in Canada, and their observations provide a perspective which, given the similarities between Canada's health care system and others in the west, may make the proposals in this text more accessible internationally. Chapter 7 presents the results of a comprehensive survey of Canadian health care ethics consultants which offers some insight into who is doing what, and how.

The book is not, however, just another collection of individual papers around a central theme. Baylis has arranged the chapters to comment on the novel and illuminating 'profile' of the health care ethics consultant, which she presents in chapter 2 . The objective of the profile is to describe 'knowledge requirements', 'requisite abilities', and 'traits of character' for the health care consultant. The list is comprehensive and instructive, providing a central and powerful heuristic throughout the text by forcing readers, irrespective of their loyalties, to question whether its constitutive components are exhaustive, appropriate and attainable. At the very least, the profile is an important tool for structuring further debate.

Methodologically, this text is far more philosophically grounded than the other; it is not so much a 'practical guide' as an extended discussion, with the profile forming the concrete proposal. In this sense it is inappropriate to compare one with the other since they are not trying to do the same thing.

Lynch's discussion of the skill and abilities which attend to the 'facilitation role' (chapter 3) probes important questions that have not been treated with depth elsewhere in the bioethics literature.

Some topics are treated in both texts, albeit differently. For example, both books discuss the work environment of ethics consultants. In their chapter 'Setting Up Practice' LaPuma and Schiedermayer identify ten issues, and in keeping with the orientation of the book, provide practical guides for what one needs to do. In one of the most enjoyable and stimulating chapters of the Baylis book (chapter 5), Benjamin Freedman provides a tem- plate and a justification for describing the 'median conditions [that] need to be specified on behalf of working conditions, established as of right, on behalf of health care ethics consultants .... More than a list of demands or expectations, it is a philosophical explanation of why it is difficult to produce an adequate job description we have not agreed on the internal morality of health care ethics consultation. But Freedman goes further and offers a reasonable description of the job. Importantly, many of the controversial issues which I suggested that LaPuma and Schiedermayer treated as uncontroversial and resolved, are challenged directly by Freedman (for example, whether consent from a physician is a prerequisite for an ethics consultation).

Legal issues are treated briefly in LaPuma and Schiedermayer as questions about the amount of malpractice coverage one ought to have (pages 79-81), whereas chapter 6 by Lowenstein and Des Brisay in the Baylis book provides a more comprehensive discussion (almost a primer) of the 'facts' relating to liability and the potential for ethics consultants and ethics committees to be found liable for damages as a result of their consultations (which they conclude is unlikely in tort law). Their review of the literature leading to this point is of interest because it is based on some assumptions about the standard of care that any health professional must hold to.

This book is not without some weaknesses. Ironically, one of the weaker chapters is that which is devoted to multidisciplinarity. In chapter 4, 'Feeder Disciplines', individuals from law, medicine, nursing, philosophy and theology provide an assessment of how well or poorly these disciplines meet the standards outlined in the Baylis profile. It is somewhat tedious to read; without the profile handy as a checklist, it is difficult to appreciate how significant are the omissions attributed to each feeder discipline in respect of the profile. It also suffers from falling somewhere between the personal accounts of these highly qualified commentators about how their discipline meets or fails to meet the criteria of the profile, and a general description of this success or failure. There are a few occasions when phrases seem to be repeated in different sections. Sixteen pages are used to provide copies of the survey instrument reported in chapter 7 , and the index is short.

Read alone, this book will suggest to readers that there is much to be gained from having comprehensive knowledge and abilities (and hopefully some of the character traits) of each of several disciplines. This might leave some depressed as they go through the profile comparing themselves to it. A more modest conclusion is that ethics consultation has now come of age as an academic discipline and a professional service. Both books challenge all who profess (or aspire) to 'work in clinical ethics' to come to terms with what this activity entails.

ERIC M MESLIN, PHD Visiting Clinical Ethicist, Oxford Radcliffe Hospital, Visiting Fellow, Green College, Oxford University

\section{Philosophical perspectives on psychiatric diagnostic classification}

Edited by John $Z$ Sadler, Osborne $P$ Wiggins and Michael A Schwartz, Baltimore and London, The Johns Hopkins University Press, 1994, 400 pages, $£ 57.50 \mathrm{hc}, £ 20.50$ sc.

In 1950, the American Psychiatric Association completed its first classificatory manual of psychiatric disorders: Diagnostic and Statistical Manual of Mental Disorders (DSM). The 1980 version (DSM-III), revised again in 1987 as DSM-III-R, is arguably the most influential psychiatric taxonomy in the world today. Among other things, it influences whether psychiatrists will call a person mad, and what kind of madness they will say he or she has.

Contributors to Philosophical Perspectives on Psychiatric Diagnostic Classification were asked to focus on one aspect of DSM, criticise it and offer an alternative. The resulting fourteen essays represent the first detailed analytical examination of the philosophical assumptions and commitments of DSM. The collection also examines the consequences of adopting different nosologies, attempts to clarify the methodological approaches to psychiatric classification and outlines some of the criteria for evaluation of various nosologies. It deals with questions such as: What is a mental disorder? What should be included in the classification of mental 